\title{
Introduction: Opportunities, Artifacts, and Interpretation of Aberration-Corrected Electron Microscopy Data
}

A one-day pre-meeting workshop was held on August 7, 2011, immediately preceding the annual Microscopy and Microanalysis (M\&M) 2011 meeting in Nashville, Tennessee. The workshop was organized by the Aberration-Corrected Electron Microscopy (ACEM) Focused Interest Group, with an Organizing Committee consisting of Phil Batson, Dave Muller, Larry Allard, Paul Voyles, Miofang Chi, and Mike O'Keefe.

The workshop was intended as a forum for the discussion of the latest advances and solutions to problems associated with application of aberration-correction technology in transmission and scanning transmission electron microscopy. The format consisted of platform presentations by both invited and contributed speakers, chosen for their potential to spark discussion, together with a working lunch-poster session. Over 100 people participated during the full-day M\&M pre-meeting. Invited speakers covered innovations and issues, while contributors (both platform and poster) highlighted practical experiences and solutions to problems encountered during the application of ACEM to on-going experimental studies. The topical areas for the workshop included new corrector designs, image collection/interpretation, and innovations in spectroscopies or new signal types, as well as artifacts and practical experiences in applications of ACEM to difficult situations such as hard/soft materials and in situ experiments. All platform presentations were intentionally kept short $(\sim 15-20$ minutes $)$ to allow the maximum amount of interaction and information flow among attendees. This special section of Microscopy and Microanalysis highlights contributions from presenters at the meeting, which went through the usual refereeing process before being accepted for publication in this issue. These papers provide but a small snapshot of the exciting new developments and opportunities for the burgeoning field of aberration-corrected electron microscopy.

Phil Batson

Co-editor

Dave Smith

Co-editor 